\title{
Inflation of Universe by Nonlinear Electrodynamics
}

\author{
S. I. Kruglov ${ }^{1}$ \\ Department of Physics, University of Toronto, \\ 60 St. Georges St., Toronto, ON M5S 1A7, Canada \\ Department of Chemical and Physical Sciences, University of Toronto, \\ 3359 Mississauga Road North, Mississauga, ON L5L 1C6, Canada
}

\begin{abstract}
Nonlinear electrodynamics with two dimensional parameters is studied. The range of electromagnetic fields when principles of causality, unitarity and the classical stability hold are obtained. A singularity of the electric field at the center of charges is absent within our model and there are corrections to the Coulomb law as $r \rightarrow \infty$. The universe inflation takes place in the background of stochastic magnetic fields. The second stage of the universe evolution is the radiation era so that the graceful exit exists. We estimated the spectral index, the tensor-to-scalar ratio, and the running of the spectral index that are in an approximate agreement with the PLANK and WMAP data.
\end{abstract}

keywords: Nonlinear electrodynamics; inflation; causality; unitarity; stochastic magnetic fields

\section{Introduction}

The universe inflation can be explained by modifying the general relativity (GR) [1], [2]. But it is possible to explain the inflation of the universe by coupling GR with nonlinear electromagnetic fields [3], [4], [5], [6], [7], [8], $[9],[10],[11],[12],[13],[14]$. In the early time of the universe evolution electromagnetic fields were very strong and quantum corrections should be taken into account [15] and, as a result, Maxwell's electrodynamics becomes nonlinear electrodynamics (NED) [16], [17], [18]. First NED, without singularities of point-like charges, was proposed by Born and Infeld [19]. In this paper we study NED that for weak fields leads to the Maxwell limit. We assume that the universe filled by the stochastic magnetic background.

Stochastic fluctuations in electron-positron plasma can lead to a stochastic magnetic field [20], [21]. Thus, in the early stage of the radiationdominated era the early universe was filled by a strong low-frequency random

\footnotetext{
${ }^{1}$ E-mail: serguei.krouglov@utoronto.ca
} 
magnetic fields. It is known that there are magnetic fields in the order of $B=10^{-6} \mathrm{G}$ in our galaxy and other spiral galaxies [22], and they possess the primordial origin. Such magnetic fields can be generated by means of the galactic dynamo mechanism. According to the galactic dynamo theory angular momentum energy is transferred into magnetic energy. This mechanism needs the existence of weak seed fields in the order of $B=10^{-19} \mathrm{G}$ at the epoch of the galaxy formation. Such seed magnetic fields may be the result of thermal fluctuations of the primordial plasma and the long wavelength fluctuations can be reconnected. Then the magnetic energy may be redistributed over larger scales. It should be noted that the origin of cosmic magnetism on the largest scales of galaxies and galaxy clusters is still an open problem [23]. We consider the magnetic background because the electric field is screened by the charged primordial plasma [21]. In the standard cosmological model the asymmetry in the direction is absent, $\langle\mathbf{B}\rangle=0$, and there is no the directional effects.

The structure of the paper is as follows. In Sect. 2 we consider a NED model with two dimensional parameters $\beta, \gamma$, and the causality and unitarity principles are studied. We analyze field equations and their dual invariance in Sect. 3. It is demonstrated that there is no singularity of the electric field at the origin of the point-like charges and the electric field possesses the maximum. There are corrections to Coulomb's law in the order of $\mathcal{O}\left(r^{-6}\right)$. We estimate the model parameters $\beta$ and $\gamma$ by the requirement that at the weak field limit our model is converted into QED with one loop correction. In Sect. 4 we investigate the cosmology of the universe which is filled by stochastic magnetic fields. The energy density and pressure as the functions of the scale factor are obtained. It was demonstrated that the singularity of the Ricci scalar is absent. The evolution of the universe is studied in Sect. 5. The dependence of the scale factor on the time is found. The bound on the speed of sound which guarantees the classical stability and causality is calculated. We evaluate in Sect. 6 the cosmological parameters (the spectral index $n_{s}$, the tensor-to-scalar ratio $r$, and the running of the spectral index $\alpha_{s}$ ). It is shown that they agreed approximately with the PLANK and WMAP data. Section 7 is a conclusion.

We use the units with $c=\hbar=\varepsilon_{0}=\mu_{0}=1$. The metric signature is chosen to be $\eta=\operatorname{diag}(-,+,+,+)$. 


\section{The NED model}

Here, we propose NED with the Lagrangian density given by

$$
\mathcal{L}=-\frac{\mathcal{F}}{(1+2 \beta \mathcal{F})^{3 / 2}}+\frac{\gamma}{2} \mathcal{G}^{2},
$$

where $\mathcal{F}=(1 / 4) F_{\mu \nu} F^{\mu \nu}=\left(\mathbf{B}^{2}-\mathbf{E}^{2}\right) / 2, \mathcal{G}=(1 / 4) F_{\mu \nu} \tilde{F}^{\mu \nu}=\mathbf{B} \cdot \mathbf{E}\left(\tilde{F}^{\mu \nu}=\right.$ $\epsilon^{\mu \nu \alpha \beta} F_{\alpha \beta} / 2$ is a dual tensor $), F_{\mu \nu}=\partial_{\mu} A_{\nu}-\partial_{\nu} A_{\mu}$, and $\beta(\beta>0), \gamma(\gamma>0)$ are dimensional parameters. The symmetrical stress-tensor following from Eq. (1) is

$$
\begin{aligned}
& T_{\mu \nu}=\mathcal{L}_{\mathcal{F}} F_{\mu}{ }^{\alpha} F_{\nu \alpha}+\frac{1}{2} \mathcal{L}_{\mathcal{G}}\left(F_{\mu}{ }^{\alpha} \tilde{F}_{\nu \alpha}+F_{\nu}{ }^{\alpha} \tilde{F}_{\mu \alpha}\right)-g_{\mu \nu} \mathcal{L} \\
= & \frac{(\beta \mathcal{F}-1) F_{\mu}{ }^{\alpha} F_{\nu \alpha}}{(1+2 \beta \mathcal{F})^{5 / 2}}+\frac{1}{2} \gamma \mathcal{G}\left(F_{\mu}^{\alpha} \tilde{F}_{\nu \alpha}+F_{\nu}{ }^{\alpha} \tilde{F}_{\mu \alpha}\right)-g_{\mu \nu} \mathcal{L},
\end{aligned}
$$

where $\mathcal{L}_{\mathcal{F}}=\partial \mathcal{L} / \partial \mathcal{F}, \mathcal{L}_{\mathcal{G}}=\partial \mathcal{L} / \partial \mathcal{G}$. Making use of Eq. (2) we find the trace of the stress-tensor

$$
\mathcal{T} \equiv T_{\mu}{ }^{\mu}=\frac{12 \beta \mathcal{F}^{2}}{(1+2 \beta \mathcal{F})^{5 / 2}}+2 \gamma \mathcal{G}^{2} .
$$

In Maxwell's electrodynamics $\beta \rightarrow 0, \gamma \rightarrow 0$ and $\mathcal{L} \rightarrow-\mathcal{F}$ so that the energymomentum tensor is traceless, $\mathcal{T} \rightarrow 0$. Because the dimensional parameters are present in the model the scale invariance is violated and the stress-tensor is not traceless. From Eq. (1) one obtains the energy density $\rho$ and the pressure $p$ as follows:

$$
\begin{gathered}
\rho=-\mathcal{L}-E^{2} \mathcal{L}_{\mathcal{F}}+\mathcal{G} \mathcal{L}_{\mathcal{G}}=\frac{(1-\beta \mathcal{F}) E^{2}}{(1+2 \beta \mathcal{F})^{5 / 2}}+\frac{\mathcal{F}}{(1+2 \beta \mathcal{F})^{3 / 2}}+\frac{1}{2} \gamma \mathcal{G}^{2} \\
p=\mathcal{L}+\frac{E^{2}-2 B^{2}}{3} \mathcal{L}_{\mathcal{F}}-\mathcal{G} \mathcal{L}_{\mathcal{G}}=-\frac{\mathcal{F}}{(2 \beta \mathcal{F}+1)^{3 / 2}}+\frac{\left(E^{2}-2 B^{2}\right)(\beta \mathcal{F}-1)}{3(2 \beta \mathcal{F}+1)^{5 / 2}}-\frac{1}{2} \gamma \mathcal{G}^{2}
\end{gathered}
$$

\subsection{The causality and unitarity principles}

In accordance with the causality principle the group velocity of excitations over the background should be less than the speed of light and there will be 
not tachyons in the theory spectrum. The unitarity principle guarantees the absence of ghosts. Both principles lead to the inequalities [24]:

$$
\begin{gathered}
\mathcal{L}_{\mathcal{F}} \leq 0, \quad \mathcal{L}_{\mathcal{F F}} \geq 0, \quad \mathcal{L}_{\mathcal{G G}} \geq 0, \\
\mathcal{L}_{\mathcal{F}}+2 \mathcal{F} \mathcal{L}_{\mathcal{F F}} \leq 0, \quad 2 \mathcal{F} \mathcal{L}_{\mathcal{G G}}-\mathcal{L}_{\mathcal{F}} \geq 0
\end{gathered}
$$

By virtue of Eq. (1) one obtains

$$
\begin{gathered}
\mathcal{L}_{\mathcal{F}}=\frac{\beta \mathcal{F}-1}{(1+2 \beta \mathcal{F})^{5 / 2}}, \quad \mathcal{L}_{\mathcal{G G}}=\gamma, \quad 2 \mathcal{F} \mathcal{L}_{\mathcal{G G}}-\mathcal{L}_{\mathcal{F}}=2 \mathcal{F} \gamma+\frac{1-\beta \mathcal{F}}{(1+2 \beta \mathcal{F})^{5 / 2}}, \\
\mathcal{L}_{\mathcal{F}}+2 \mathcal{F} \mathcal{L}_{\mathcal{F F}}=\frac{-4(\beta \mathcal{F})^{2}+11 \beta \mathcal{F}-1}{(1+2 \beta \mathcal{F})^{7 / 2}}, \quad \mathcal{L}_{\mathcal{F F}}=\frac{3 \beta(2-\beta \mathcal{F})}{(1+2 \beta \mathcal{F})^{7 / 2}} .
\end{gathered}
$$

Making use of Eqs. (6) and (7), in the case of $\gamma=0, \mathbf{B}=0$, we obtain $|\mathbf{E}| \leq \sqrt{1 / \beta}$ which is satisfied because the maximum of the electric field is given by $\left|\mathbf{E}_{\max }\right|=\sqrt{1 / \beta}$ (see Eq. (19)). If $\gamma=0, \mathbf{E}=0$, one has $|\mathbf{B}| \leq \sqrt{(11-\sqrt{105}) /(4 \beta)} \approx 0.434 / \sqrt{\beta}$.

\section{Electromagnetic field equations}

With the help of Eq. (1) we find field equations

$$
\partial_{\mu}\left(\mathcal{L}_{\mathcal{F}} F^{\mu \nu}+\mathcal{L}_{\mathcal{G}} \tilde{F}^{\mu \nu}\right)=0
$$

Making use of Eqs. (1) and (8) we obtain

$$
\partial_{\mu}\left(\frac{(\beta \mathcal{F}-1) F^{\mu \nu}}{(1+2 \beta \mathcal{F})^{5 / 2}}+\gamma \mathcal{G} \tilde{F}^{\mu \nu}\right)=0 .
$$

Using the equation $\mathbf{D}=\partial \mathcal{L} / \partial \mathbf{E}$, we find the electric displacement field

$$
\mathbf{D}=\frac{1-\beta \mathcal{F}}{(1+2 \beta \mathcal{F})^{5 / 2}} \mathbf{E}+\gamma \mathcal{G} \mathbf{B} .
$$

The magnetic field $\mathbf{H}=-\partial \mathcal{L} / \partial \mathbf{B}$ is given by

$$
\mathbf{H}=\frac{1-\beta \mathcal{F}}{(1+2 \beta \mathcal{F})^{5 / 2}} \mathbf{B}-\gamma \mathcal{G} \mathbf{E}
$$


We use the decomposition of Eqs. (10) and (11) as follows [25]:

$$
D_{i}=\varepsilon_{i j} E^{j}+\nu_{i j} B^{j}, \quad H_{i}=\left(\mu^{-1}\right)_{i j} B^{j}-\nu_{j i} E^{j} .
$$

Making use of Eqs. (10), (11) and (12) one finds

$$
\begin{gathered}
\varepsilon_{i j}=\delta_{i j} \varepsilon, \quad\left(\mu^{-1}\right)_{i j}=\delta_{i j} \mu^{-1}, \quad \nu_{j i}=\delta_{i j} \nu, \\
\varepsilon=\frac{1-\beta \mathcal{F}}{(1+2 \beta \mathcal{F})^{5 / 2}}, \quad \mu^{-1}=\varepsilon=\frac{1-\beta \mathcal{F}}{(1+2 \beta \mathcal{F})^{5 / 2}}, \quad \nu=\gamma \mathcal{G} .
\end{gathered}
$$

Field equation (9), by virtue of Eqs. (10) and (11), can be represented as the Maxwell equations

$$
\nabla \cdot \mathbf{D}=0, \quad \frac{\partial \mathbf{D}}{\partial t}-\nabla \times \mathbf{H}=0
$$

Because $\varepsilon_{i j},\left(\mu^{-1}\right)_{i j}$, and $\nu_{j i}$ depend on electromagnetic fields, Eq. (14) are nonlinear Maxwell's equations. Using the Bianchi identity $\partial_{\mu} \tilde{F}^{\mu \nu}=0$, we obtain the second pair of Maxwell's equations

$$
\nabla \cdot \mathbf{B}=0, \quad \frac{\partial \mathbf{B}}{\partial t}+\nabla \times \mathbf{E}=0 .
$$

With the aid Eqs. (10) and (11) we find

$$
\mathbf{D} \cdot \mathbf{H}=\left(\varepsilon^{2}-\nu^{2}\right) \mathbf{E} \cdot \mathbf{B}+\varepsilon \nu\left(\mathbf{B}^{2}-\mathbf{E}^{2}\right) .
$$

The dual symmetry is broken as $\mathbf{D} \cdot \mathbf{H} \neq \mathbf{E} \cdot \mathbf{B}[26]$. In BI electrodynamics and in Maxwell's electrodynamics $(\varepsilon=1, \nu=0)$ the dual symmetry holds. In quantum electrodynamics with quantum corrections and in generalized BI electrodynamics [27] the dual symmetry is violated.

\subsection{The fields of point-like electric charges}

When the source is a point-like electric charge $q_{e}$, the electric displacement field obeys the equation

$$
\nabla \cdot \mathbf{D}=4 \pi q_{e} \delta(\mathbf{r})
$$

Making use of Eq. (10) at $\mathbf{B}=0$ the solution to Eq. (17) is given by

$$
\frac{E\left(2+\beta E^{2}\right)}{2\left(1-\beta E^{2}\right)^{5 / 2}}=\frac{q_{e}}{r^{2}}
$$


In accordance with Eq. (18) as $r \rightarrow 0$ the solution is

$$
E(0)=\sqrt{\frac{1}{\beta}} .
$$

Equation (19) shows the maximum of the electric field in the center of charged particles similar to BI electrodynamics. Thus, at the origin of the pointlike charges there is no singularity of the electric field unlike the Maxwell electrodynamics. Let us introduce unitless variables

$$
x=\frac{r^{2}}{q_{e} \sqrt{\beta}}, \quad y=\sqrt{\beta} E .
$$

Then Eq. (18) can be written as follows:

$$
\frac{y\left(2+y^{2}\right)}{2\left(1-y^{2}\right)^{5 / 2}}=\frac{1}{x} .
$$

The function $y(x)$ is depicted in Fig. 1. The approximate real and positive

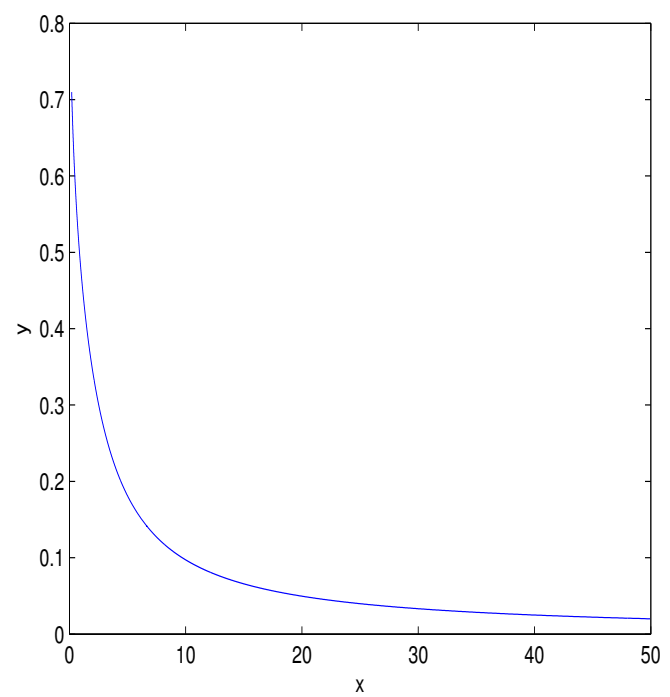

Figure 1: The function $y(x)$.

solutions to Eq. (21) are in Table 1. The function $y(x)$ as $x \rightarrow \infty$ has the 


\section{Table 1:}

\begin{tabular}{ccccccccccc}
\hline$x$ & 1 & 2 & 3 & 4 & 5 & 6 & 7 & 8 & 9 & 10 \\
\hline$y$ & 0.475 & 0.344 & 0.267 & 0.217 & 0.181 & 0.155 & 0.135 & 0.120 & 0.107 & 0.097 \\
\hline
\end{tabular}

asymptotic

$$
y=\frac{1}{x}-\frac{3}{x^{3}}+\mathcal{O}\left(x^{-5}\right) .
$$

Making use of Eqs. (20) and (22) we obtain the electric field as $r \rightarrow \infty$

$$
E(r)=\frac{q_{e}}{r^{2}}-\frac{3 \beta q_{e}^{3}}{r^{6}}+\mathcal{O}\left(r^{-10}\right)
$$

According to Eq. (23) Coulomb's law possesses corrections in the order of $\mathcal{O}\left(r^{-6}\right)$. At $\beta=0$ one finds the Coulomb law $E=q_{e} / r^{2}$ similar to Maxwell's electrodynamics. Making use of Eq. (21), one finds the asymptotic of $y(x)$ as $x \rightarrow 0$

$$
y(x)=1-0.59 x^{0.4} \quad x \rightarrow 0 .
$$

With the help of Eq. (20), we obtain the asymptotic

$$
E(r)=\frac{1}{\sqrt{\beta}}-\frac{0.59 r^{0.8}}{q_{e}^{0.4} \beta^{0.7}} \quad r \rightarrow 0 .
$$

Equation (25) at $r=0$ leads to Eq. (19) and gives the electric field over short distances.

\subsection{Estimation of parameters $\beta$ and $\gamma$}

Now, we define the model parameters $\beta$ and $\gamma$ by the requirement that at the weak field limit our model is converted into the Heisenberg-Euler electrodynamics. Expanding Lagrangian (1) at small value $\beta \mathcal{F} \ll 1$, we have

$$
\mathcal{L}=-\mathcal{F}+3 \beta \mathcal{F}^{2}-\frac{15}{2} \beta^{2} \mathcal{F}^{3}+\mathcal{O}\left((\beta \mathcal{F})^{4}\right)+\frac{\gamma}{2} \mathcal{G}^{2}
$$

The QED Lagrangian with one loop correction (the Heisenberg-Euler Lagrangian) is given by [28]

$$
\mathcal{L}_{H E}=-\mathcal{F}+c_{1} \mathcal{F}^{2}+c_{2} \mathcal{G}^{2}, \quad c_{2}=\frac{14 \alpha^{2}}{45 m_{e}^{4}}, \quad c_{1}=\frac{8 \alpha^{2}}{45 m_{e}^{4}},
$$


where the coupling constant $\alpha=e^{2} /(4 \pi) \approx 1 / 137$ and the electron mass $m_{e}=0.51 \mathrm{MeV}$. By identifying Eqs. (26) and (27) we obtain

$$
\beta=\frac{8 \alpha^{2}}{135 m_{e}^{4}}=4.6 \times 10^{-5} \mathrm{MeV}^{-4}, \quad \gamma=\frac{28 \alpha^{2}}{45 m_{e}^{4}}=4.9 \times 10^{-4} \mathrm{MeV}^{-4} \text {. }
$$

\section{Cosmology}

The action of GR coupled to electromagnetic fields is given by

$$
S=\int d^{4} x \sqrt{-g}\left[\frac{1}{2 \kappa^{2}} R+\mathcal{L}\right]
$$

where $M_{P l}=\kappa^{-1}$ is the reduced Planck mass and $R$ is the Ricci scalar. By varying the action (29) we obtain the Einstein and electromagnetic field equations

$$
\begin{gathered}
R_{\mu \nu}-\frac{1}{2} g_{\mu \nu} R=-\kappa^{2} T_{\mu \nu}, \\
\partial_{\mu}\left(\frac{\sqrt{-g} F^{\mu \nu}(\beta \mathcal{F}-1)}{(2 \beta \mathcal{F}+1)^{5 / 2}}\right)=0 .
\end{gathered}
$$

The squared of the line element of homogeneous and isotropic spacetime is given by

$$
d s^{2}=-d t^{2}+a(t)^{2}\left(d x^{2}+d y^{2}+d z^{2}\right)
$$

$a(t)$ is a scale factor. We assume that the cosmic background filled by stochastic magnetic fields. The averaged magnetic fields (that are sources of gravitational fields) which guaranty the isotropy of the Friedman-Robertson-Walker (FRW) spacetime obey the equations [29]

$$
\langle\mathbf{B}\rangle=0, \quad\left\langle E_{i} B_{j}\right\rangle=0, \quad\left\langle B_{i} B_{j}\right\rangle=\frac{1}{3} B^{2} g_{i j} .
$$

Here, the brackets $\langle$.$\rangle mean an average over a volume but for a simplicity$ in the following we will omit the brackets $\langle$.$\rangle . The NED energy-momentum$ tensor with Eqs. (33) represents a perfect fluid [8]. The Friedman equation is given as follows:

$$
3 \frac{\ddot{a}}{a}=-\frac{\kappa^{2}}{2}(\rho+3 p),
$$

where $\dot{a}(t)=\partial a / \partial t$, so that the dot under the letter means the derivative with respect to the cosmic time. When $\rho+3 p<0$ we have $\ddot{a}>0$ and the 
universe acceleration holds. According to the standard cosmological model, an isotropic symmetry is guarantied if $\langle\mathbf{B}\rangle=0$. Making use of Eqs. (4) and (5) we find (in the case of $\mathbf{E}=0$ )

$$
\rho+3 p=-\frac{B^{2}\left(2 \beta B^{2}-1\right)}{\left(1+\beta B^{2}\right)^{5 / 2}} .
$$

The plot of $\beta(\rho+3 p)$ as a function of $\beta B^{2}$ is represented by Fig. 2. If

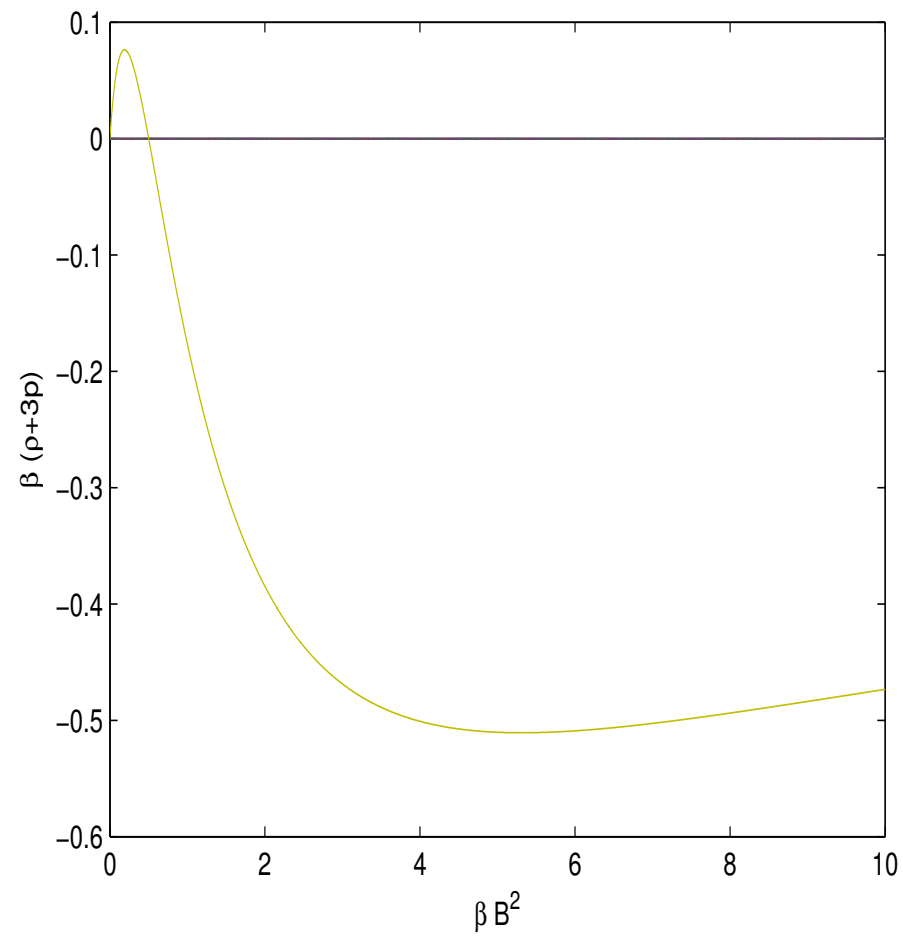

Figure 2: The function $\beta(\rho+3 p)$ versus $\beta B^{2}$.

$\beta B^{2}>0.5$ one has $\rho+3 p<0$ and the universe acceleration occurs. As a result, the strong magnetic fields lead to the inflation of the universe. Consider the conservation of the stress-tensor, $\nabla^{\mu} T_{\mu \nu}=0$,

$$
\dot{\rho}+3 \frac{\dot{a}}{a}(\rho+p)=0
$$


With the aid of Eqs. (4) and (5) if $\mathbf{E}=0$, one obtains

$$
\rho=\frac{B^{2}}{2\left(1+\beta B^{2}\right)^{3 / 2}}, \quad \rho+p=\frac{B^{2}\left(2-\beta B^{2}\right)}{3\left(1+\beta B^{2}\right)^{5 / 2}} .
$$

Taking into account Eq. (37), one finds the solution to Eq. (36)

$$
B(t)=\frac{B_{0}}{a^{2}(t)}
$$

where $B_{0}$ is the value of the magnetic field which corresponds to $a(t)=1$. The scale factor $a(t)$ increases due to the universe expansion and the magnetic field $B(t)$ decreases. With the help of Eqs. (37) and (38) we obtain the energy density and pressure

$$
\rho(t)=\frac{a^{2}(t) B_{0}^{2}}{2\left(a^{4}(t)+\beta B_{0}^{2}\right)^{3 / 2}}, \quad p(t)=\frac{a^{2}(t) B_{0}^{2}\left(a^{4}(t)-5 \beta B_{0}^{2}\right)}{6\left(a^{4}(t)+\beta B_{0}^{2}\right)^{5 / 2}} .
$$

Making use of Eqs. (39) one finds

$$
\lim _{a(t) \rightarrow 0} \rho(t)=\lim _{a(t) \rightarrow 0} p(t)=\lim _{a(t) \rightarrow \infty} \rho(t)=\lim _{a(t) \rightarrow \infty} p(t)=0 .
$$

Equation (40) shows that there are not singularities of the density of the energy and pressure as $a(t) \rightarrow 0$ and $a(t) \rightarrow \infty$. The plot of the equation of state $(\operatorname{EoS}) w=p(t) / \rho(t)$ versus $x=a(t) /\left(\beta B_{0}^{2}\right)^{1 / 4}$ is depicted in Fig. 3 . Making use of Eqs. (39) we obtain

$$
\lim _{x \rightarrow \infty} w=\lim _{x \rightarrow \infty} \frac{x^{4}-5}{3\left(x^{4}+1\right)}=\frac{1}{3} .
$$

In accordance with Eq. (41) the EoS corresponds to the ultra-relativistic behaviour [30] as $a(t) \rightarrow \infty$. The de Sitter spacetime, $w=-1$, is realized for $x=1 / \sqrt[4]{2} \approx 0.84$. From Eq. (3) and (30) we find the Ricci scalar

$$
R=\kappa^{2} \mathcal{T}=\frac{3 \kappa^{2} \beta B^{4}}{\left(1+\beta B^{2}\right)^{5 / 2}}=\kappa^{2}[\rho(t)-3 p(t)] .
$$

The $\beta R / \kappa^{2}$ as a function of $\left[1 /\left(\beta B_{0}^{2}\right)\right]^{1 / 4} a$ is depicted in Fig. 4. Making use of Eqs. (40) and (42) one finds

$$
\lim _{a(t) \rightarrow 0} R(t)=\lim _{a(t) \rightarrow \infty} R(t)=0
$$




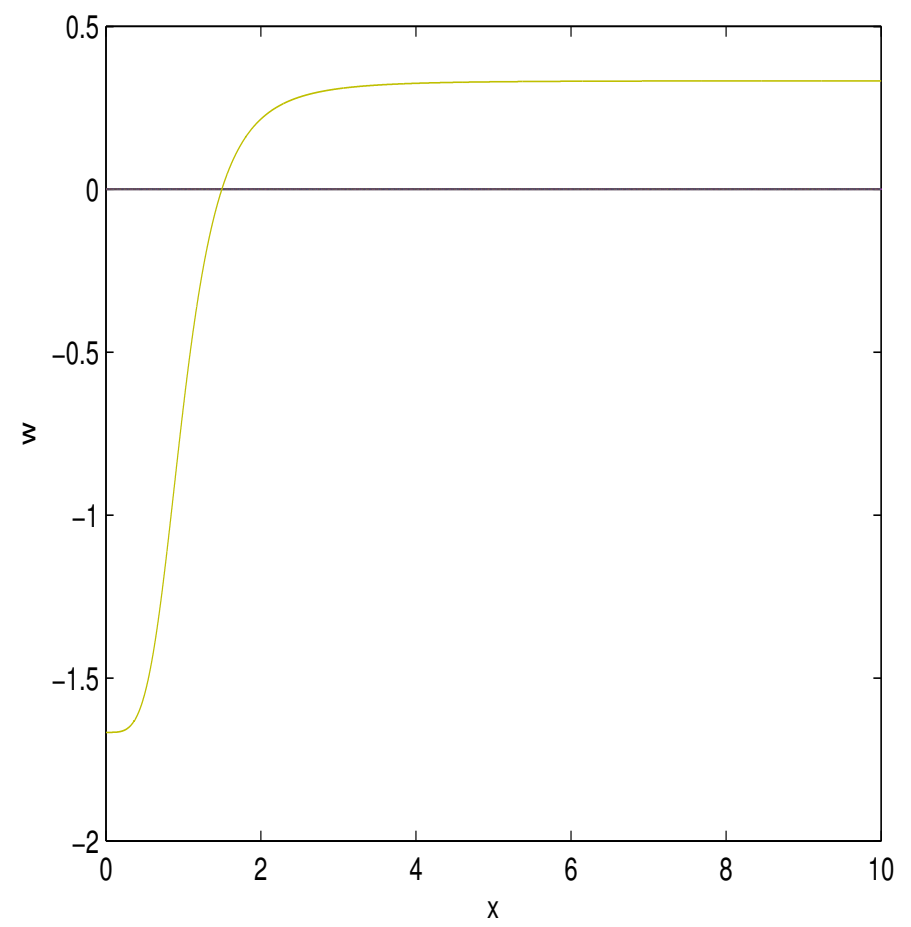

Figure 3: The function $w$ versus $x=a /\left(\beta B_{0}^{2}\right)^{1 / 4}$.

In accordance with Eq. (43) there is not a singularity of the Ricci scalar. The Kretschmann scalar $R_{\mu \nu \alpha \beta} R^{\mu \nu \alpha \beta}$ and the Ricci tensor squared $R_{\mu \nu} R^{\mu \nu}$ can be expressed in the form of combinations of $\kappa^{4} \rho^{2}, \kappa^{4} \rho p$, and $\kappa^{4} p^{2}$ [11]. Then they vanish, in accordance with Eq. (40), as $a(t) \rightarrow 0$ and $a(t) \rightarrow \infty$. During the evolution of the universe the scale factor increases as $t \rightarrow \infty$ and spacetime becomes flat. Equations (35) and (38) show that the universe acceleration takes place at $a(t)<(2 \beta)^{1 / 4} \sqrt{B_{0}} \approx 1.19 \beta^{1 / 4} \sqrt{B_{0}}$.

\section{Evolution of the universe}

For the three dimensional flat universe the second Friedman equation is

$$
\left(\frac{\dot{a}}{a}\right)^{2}=\frac{\kappa^{2} \rho}{3} .
$$




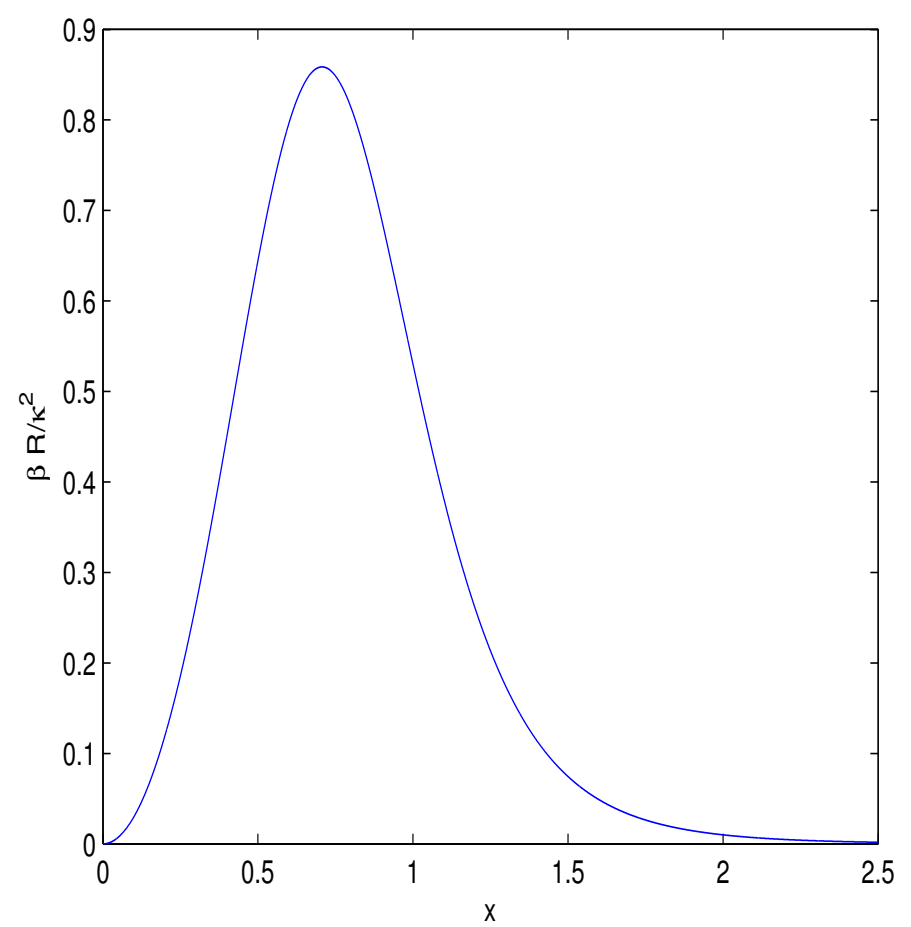

Figure 4: The function $\beta R / \kappa^{2}$ versus $x \equiv a /\left(\beta B_{0}^{2}\right)^{1 / 4}$.

Making use of Eqs. (37), (38) and (44), one obtains

$$
\dot{a}=\frac{\kappa B_{0} a^{2}}{\sqrt{6}\left(a^{4}+\beta B_{0}^{2}\right)^{3 / 4}} .
$$

Introducing the unitless variable $x=a /\left(\beta^{1 / 4} \sqrt{B_{0}}\right)$, Eq. (45) is rewritten as

$$
\dot{x}=\frac{\kappa x^{2}}{\sqrt{6 \beta}\left(x^{4}+1\right)^{3 / 4}} .
$$

The plot of the function $y \equiv \sqrt{6 \beta} \dot{x} / \kappa$ is depicted in Fig. 5. According to Fig. 5 at the initial time the universe inflation takes place, $(\dot{y}>0)$, then the graceful exit occurs at the point $x=\sqrt[4]{2}(\dot{y}=0)$ and after the universe decelerates. From Eq. (46) we obtain

$$
\int_{\epsilon}^{x} \frac{\left(x^{4}+1\right)^{3 / 4}}{x^{2}} d x=\frac{\kappa}{\sqrt{6 \beta}} \int_{0}^{t} d t .
$$




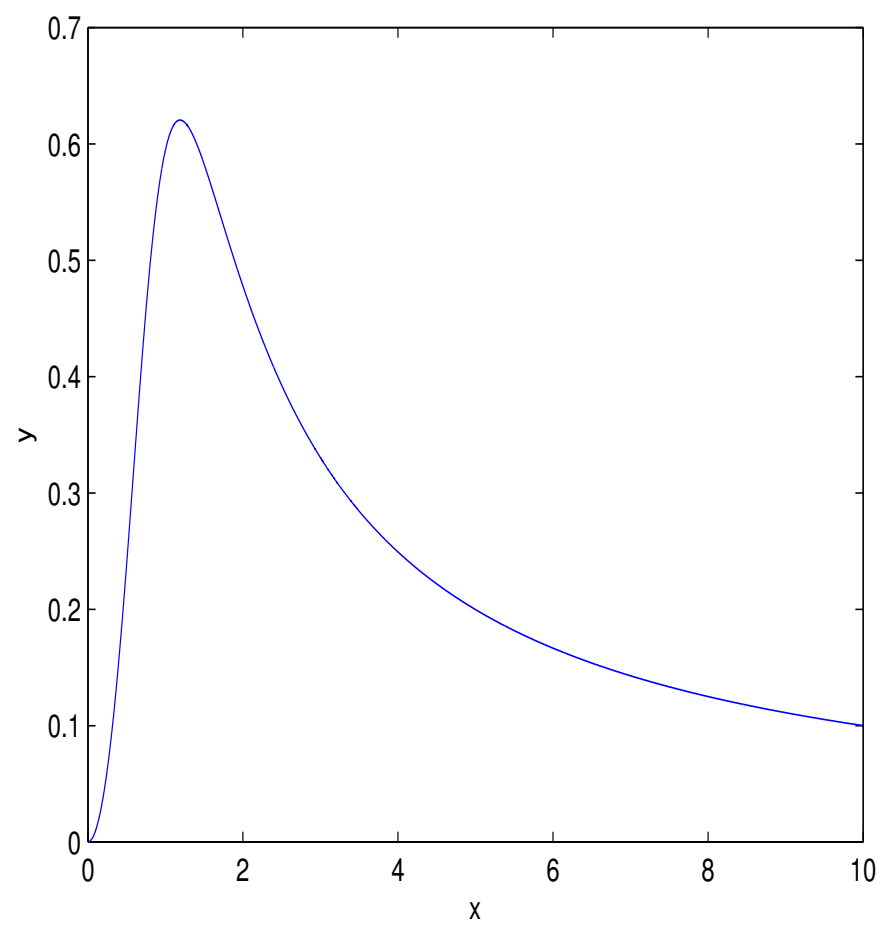

Figure 5: The function $y \equiv \sqrt{6 \beta} \dot{x} / \kappa$ versus $x$.

Calculating the integrals in Eq. (47) one arrives at

$$
\begin{gathered}
x_{2}^{3} F_{1}\left(\frac{1}{4}, \frac{3}{4} ; \frac{7}{4} ;-x^{4}\right)-\frac{\left(x^{4}+1\right)^{3 / 4}}{x} \\
-\epsilon_{2}^{3} F_{1}\left(\frac{1}{4}, \frac{3}{4} ; \frac{7}{4} ;-\epsilon^{4}\right)+\frac{\left(\epsilon^{4}+1\right)^{3 / 4}}{\epsilon}=\frac{\kappa t}{\sqrt{6 \beta}},
\end{gathered}
$$

${ }_{2} F_{1}(a, b ; c ; z)$ is the hypergeometric function, $\epsilon$ corresponds to the beginning of the universe inflation. We can study the evolution of the universe inflation from Eq. (48). To obtain the asymptotic of the scale factor as $t \rightarrow \infty$ we explore the relation [32]

$$
\begin{gathered}
{ }_{2} F_{1}(a, b ; c ; z)=\frac{\Gamma(c) \Gamma(b-a)}{\Gamma(b) \Gamma(c-a)}(-z)^{-a}{ }_{2} F_{1}(a, 1-c+a ; 1-b+a ; 1 / z) \\
+\frac{\Gamma(c) \Gamma(a-b)}{\Gamma(a) \Gamma(c-b)}(-z)^{-b}{ }_{2} F_{1}(b, 1-c+b ; 1-a+b ; 1 / z) .
\end{gathered}
$$


Making use of Eq. (49) one obtains

$$
\begin{gathered}
{ }_{2} F_{1}\left(1 / 4,3 / 4 ; 7 / 4 ;-x^{4}\right)=\frac{\Gamma(7 / 4) \Gamma(1 / 2)}{\Gamma(3 / 4) \Gamma(3 / 2)}(x)-{ }_{2} F_{1}\left(1 / 4,-1 / 2 ; 1 / 2 ;-1 / x^{4}\right) \\
+\frac{\Gamma(7 / 4) \Gamma(-1 / 2)}{\Gamma(1 / 4) \Gamma(1)}(x)_{2}^{-3} F_{1}\left(3 / 4,0 ; 3 / 2 ;-1 / x^{4}\right) .
\end{gathered}
$$

Expanding the hypergeometric functions in $1 / x^{4} \rightarrow 0$ in the leading order, we find from Eq. (48) $1.66 x^{2} \approx \kappa t / \sqrt{6 \beta}$ and the scale factor is given by

$$
a(t) \approx 0.5 \sqrt{\kappa B_{0} t} \quad t \rightarrow \infty .
$$

Equation (51) shows that the behavior of the scale factor as $t \rightarrow \infty$ corresponds to the radiation era. Let us consider the deceleration parameter, making use of Eqs. (34), (37), (38) and (44)

$$
q=-\frac{\ddot{a} a}{(\dot{a})^{2}}=\frac{x^{4}-2}{x^{4}+1} .
$$

In Fig. (6) the deceleration parameter $q$ versus $x=a /\left(\beta B_{0}^{2}\right)^{1 / 4}$ is depicted. The inflation $(q<0)$ occurs until the graceful exit point $x=\sqrt[4]{2} \approx 1.19$. The deceleration parameter becomes zero at $x=\sqrt[4]{2}$ and then the deceleration phase $(q>0)$ takes place. The similar behavior of the scale factor occurs in another model proposed in [14].

Now we estimate the amount of the inflation using the definition of efolding [33]

$$
N=\ln \frac{a\left(t_{\text {end }}\right)}{a\left(t_{\text {in }}\right)},
$$

where $t_{\text {in }}$ is an initial time and $t_{\text {end }}$ is the final time of the inflation. Using the graceful exit point $x \approx 1.19$ one obtains $a\left(t_{\text {end }}\right) \approx 1.19 b\left(b \equiv \beta^{1 / 4} \sqrt{B_{0}}\right)$. It is known that the horizon and flatness problems may be solved when e-folding is $N \approx 70$ [33]. From Eq. (53) we obtain the scale factor corresponding to the initial time of the inflation

$$
a\left(t_{i n}\right)=\frac{1.19 b}{\exp (70)} \approx 4.7 \times 10^{-31} b .
$$

Then $\epsilon \approx 4.7 \times 10^{-31}$. We use the units $\kappa=\sqrt{8 \pi G}=4.1 \times 10^{-28} \mathrm{eV}^{-1}$, $\beta=4.6 \times 10^{-29} \mathrm{eV}^{-4}$ (see subsection 3.2), $1 \mathrm{~s}=1.5 \times 10^{15} \mathrm{eV}^{-1}$ to calculate 


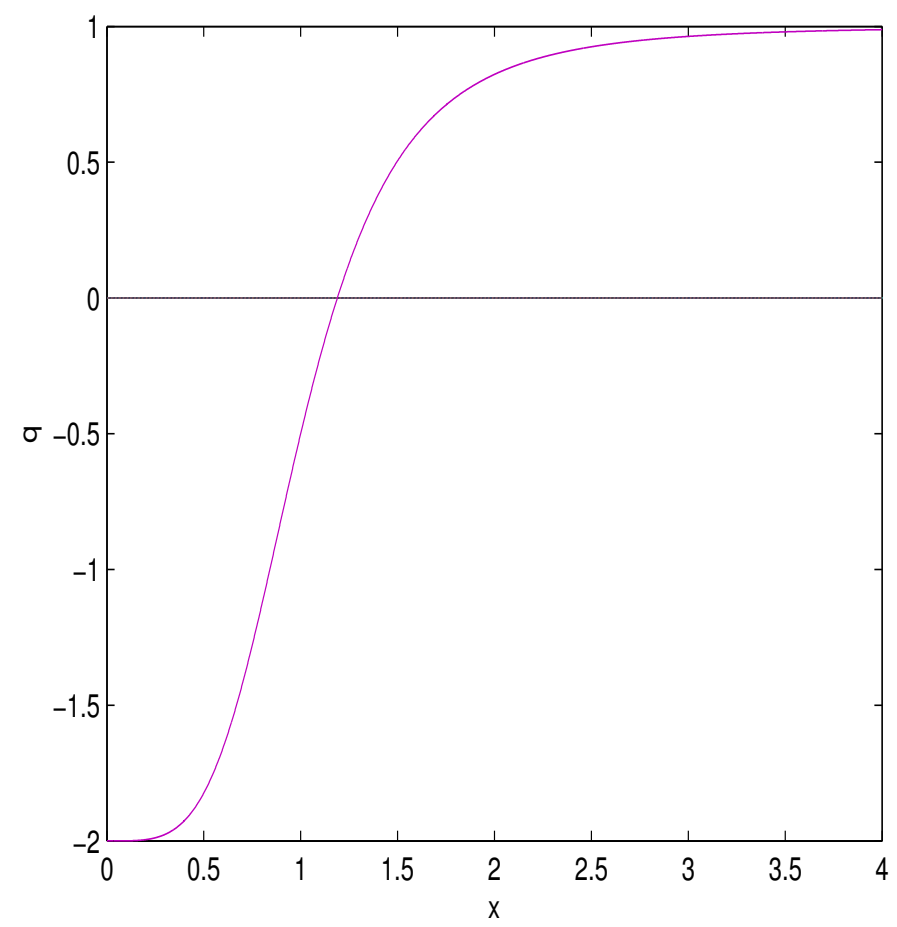

Figure 6: The function $q$ versus $x=a /\left(\beta B_{0}^{2}\right)^{1 / 4}$.

the duration of the inflationary period. Then one obtains $\kappa / \sqrt{6 \beta}=2.47 \times$ $10^{-14} \mathrm{eV}=37 \mathrm{~s}^{-1}$. Using the value $x=\sqrt[4]{2} \approx 1.19$, which corresponds to the end of the inflation, the duration of the universe inflation, according to Eq. (48), will be huge. As a result, the universe inflation will be almost eternal. Therefore, it is difficult to describe other epochs. If we use the time duration $1 \mathrm{~s}$ Eq. (47) results the value $\epsilon=0.0267$ for $x \approx 1.19$. Then the e-folding number (53) becomes $N \approx 3.8$ that is small for solving the horizon and flatness problems. One can vary the initial time $\epsilon$ and to analyze different scenarios of the universe inflation, the e-folding number, and the duration of the inflation. Thus, there are phases of the universe acceleration, deceleration and the graceful exit that are the attractive property of the model under consideration. 


\subsection{The speed of sound and causality}

The causality holds when the speed of the sound is less than the local light speed, $c_{s} \leq 1$ [31]. If the square sound speed is positive $\left(c_{s}^{2}>0\right)$ a classical stability is guarantied. From Eqs. (4) and (5) one can obtain the sound speed squared (for the case of $E=0$ )

$$
c_{s}^{2}=\frac{d p}{d \rho}=\frac{\left.5 \beta^{2} B^{4}-23 \beta B^{2}+2\right)}{3\left(\beta B^{2}+1\right)\left(2-\beta B^{2}\right)} .
$$

The classical stability requirement $c_{s}^{2}>0$ gives the bound

$$
0<\beta B^{2}<\frac{23-\sqrt{489}}{10} \approx 0.09 \text { or } 2<\beta B^{2}<\frac{23+\sqrt{489}}{10} \approx 4.5 .
$$

The causality $c_{s}^{2} \leq 1$ requires that

$$
0 \leq \beta B^{2}<2 \text { or } \beta B^{2} \geq \frac{13+\sqrt{201}}{8} \approx 3.4 .
$$

Equations (56) and (57) give the bounds

$$
0 \leq \beta B^{2}<\frac{23-\sqrt{489}}{10} \text { or } \frac{13+\sqrt{201}}{8} \leq \beta B^{2}<\frac{23+\sqrt{489}}{10}
$$

The principles of causality and unitarity studied in Sec. 2, and Eqs. (58) take place when the inequality is satisfied at the deceleration phase of the universe evolution:

$$
0 \leq \beta B^{2}<\frac{23-\sqrt{489}}{10} \approx 0.09 \text {. }
$$

The acceleration phase is realized at $\beta B^{2}>0.5$ and the classical stability, causality and unitarity are violated in this phase.

\section{The cosmological parameters}

From Eqs. (4) and (5) at $\mathbf{E}=0$ we obtain the equations

$$
p=-\rho+\frac{2 \rho\left(2-\beta B^{2}\right)}{3\left(\beta B^{2}+1\right)},
$$




$$
4 \rho^{2}\left(\beta B^{2}+1\right)^{3}-B^{4}=0 .
$$

One can find the solution (for $\beta B^{2}$ as a function of $\beta \rho$ ) to the cubic equation (61) and to place it to Eq. (60) obtaining the equation of state for perfect fluid

$$
p=-\rho+f(\rho) .
$$

If the condition $|f(\rho) / \rho| \ll 1$ is satisfied the expressions for the spectral index $n_{s}$, the tensor-to-scalar ratio $r$, and the running of the spectral index $\alpha_{s}=d n_{s} / d \ln k$ are [34]

$$
n_{s} \approx 1-6 \frac{f(\rho)}{\rho}, \quad r \approx 24 \frac{f(\rho)}{\rho}, \quad \alpha_{s} \approx-9\left(\frac{f(\rho)}{\rho}\right)^{2} .
$$

From Eqs. (63) we find the equation

$$
r=4\left(1-n_{s}\right)=8 \sqrt{-\alpha_{s}}
$$

In accordance with the PLANCK experiment [35] and WMAP data [36], [37] we have the result

$$
\begin{gathered}
n_{s}=0.9603 \pm 0.0073(68 \% C L), \quad r<0.11(95 \% C L), \\
\alpha_{s}=-0.0134 \pm 0.0090(68 \% C L) .
\end{gathered}
$$

When we take $r=0.13$, using Eqs. (65), the values for the spectral index is $n_{s}=0.9675$ and the running of the spectral index is $\alpha_{s}=-2.64 \times 10^{-4}$. Using Eq. (63) one obtains the value $f(\rho) / \rho \approx 0.005$ and from Eq. (60) the relation is

$$
\frac{f(\rho)}{\rho}=\frac{2\left(2-\beta B^{2}\right)}{3\left(\beta B^{2}+1\right)} .
$$

Then from Eq. (66) we find the value (for $r=0.13$ ) of the magnetic field $B \approx 1.4 / \sqrt{\beta} \approx 206 \mathrm{MeV}^{2} \approx 10^{12} \mathrm{~T}$ (see subsection 3.2 ) that corresponds to the inflation phase with the maximum of the energy density $\beta \rho \approx 0.192$.

\section{Conclusion}

We have considered a NED model where a singularity of the electric field in the center of charges is absent similar to BI electrodynamics. The principles of causality, the classical stability and unitarity were studied. The 
range of electromagnetic fields when causality, the classical stability and unitarity hold, were obtained. The dual symmetry is broken in this model because of dimensional parameters $\beta$ and $\gamma$. It was shown that corrections to Coulomb's law are in the order of $\mathcal{O}\left(r^{-6}\right)$. The magnetic universe with a stochastic background $\left\langle B^{2}\right\rangle \neq 0$ was studied and we demonstrated that the model with homogeneous and isotropic cosmology describes the universe inflation. There are no singularities of the energy density, pressure, the Ricci scalar, the Ricci tensor squared, and the Kretschmann scalar in our model. A stochastic magnetic field is the source of the universe inflation at the early epoch. At $B<1 / \sqrt{2 \beta}$ the universe decelerates approaching to the radiation era. The spectral index, the tensor-to-scalar ratio, and the running of the spectral index calculated are approximately in agreement with the PLANK and WMAP data. The attractive feature in our model of inflation that there is the graceful exit.

\section{References}

[1] S. Capozziello and V. Faraoni, Beyond Einstein Gravity: A Survey of Gravitational Theories for Cosmology and Astrophysics (Springer, New York, 2011).

[2] S. Nojiri and S. D. Odintsov, Phys. Rep. 505, 59 (2011) [arXiv:1011.0544].

[3] R. García-Salcedo and N. Breton, Int. J. Mod. Phys. A 15, 4341 (2000) [arXiv:gr-qc/0004017].

[4] C. S. Camara, M. R. de Garcia Maia, J. C. Carvalho, and J. A. S. Lima, Phys. Rev. D 69, 123504 (2004) [arXiv:astro-ph/0402311].

[5] E. Elizalde, J. E. Lidsey, S. Nojiri, and S. D. Odintsov, Phys. Lett. B 574, 1 (2003) [arXiv:hep-th/0307177].

[6] V. A. De Lorenci, R. Klippert, M. Novello, and J. S. Salim, Phys. Rev. D 65, 063501 (2002).

[7] M. Novello, S. E. Perez Bergliaffa, and J. M. Salim, Phys. Rev. D 69, 127301 (2004) [arXiv:astro-ph/0312093]. 
[8] M. Novello, E. Goulart, J. M. Salim, and S. E. Perez Bergliaffa, Class. Quant. Grav. 24, 3021 (2007) [arXiv:gr-qc/0610043].

[9] D. N. Vollick, Phys. Rev. D 78, 063524 (2008) [arXiv:0807.0448].

[10] R. García-Salcedo, T. Gonzalez, A. Horta-Rangel, and I. Quiros. Phys. Rev. D 90, 128301 (2014) [arXiv:1310.3021].

[11] S. I. Kruglov, Phys. Rev. D 92, 123523 (2015) [arXiv:1601.06309].

[12] S. I. Kruglov, Int.J.Mod.Phys. A 31, 1650058 (2016) [arXiv:1607.03923].

[13] S. I. Kruglov, Int.J.Mod.Phys. D 25, 1640002 (2016) [arXiv:1603.07326].

[14] S. I. Kruglov, Int.J.Mod.Phys. A32 , 1750071 (2017) [arXiv:1705.01455].

[15] J. D. Jackson, Classical Electrodynamics, 2nd edn. (Wiley, New York, 1975).

[16] W. Heisenberg and E. Euler, Z. Phys. 98, 714 (1936).

[17] J. Schwinger, Phys. Rev. 82, 664 (1951).

[18] S. L. Adler, Ann. Phys. (N.Y.) 67, 599 (1971).

[19] M. Born and L. Infeld, Proc. R. Soc. (Lond.) A 144, 425 (1934).

[20] D. Lemoine, Phys. Rev. D 51, 2677 (1995).

[21] D. Lemoine and M. Lemoine, Phys. Rev. D 52, 1955 (1995).

[22] P. P. Kronberg, Rept. Prog. Phys. 57, 325 (1994).

[23] B. M. Gaensler, R. Beck, and L. Feretti, New Astron. Rev., 48, 1003 (2004).

[24] A. E. Shabad and V. V. Usov, Phys. Rev. D 83, 105006 (2011) [arXiv:1101.2343].

[25] F. W. Hehl and Yu. N. Obukhov, Foundations of classical electrodynamics: Chage, Flux, and Metric (Birkhäuser, Boston, 2003).

[26] G. W. Gibbons and D. Rasheed, Nucl. Phys. B 454 (1995) 185. 
[27] S. I. Kruglov, J. Phys. A 43, 375402 (2010) [arXiv:0909.1032].

[28] W. Ditrich and H. Gies, Probing the Quantum Vacuum, Springer Tracts in Modern Physics, vol.166 (Springer, Berlin, 2000).

[29] R. Tolman and P. Ehrenfest, Phys. Rev. 36, 1791 (1930).

[30] L. D. Landau and E. M. Lifshits, The Classical Theory of Fields (Pergamon Press, Oxford, 1975).

[31] R. García-Salcedo, T. Gonzalez, and I. Quiros, Phys. Rev. D 89, 084047 (2014) [arXiv:1312.3163].

[32] Handbook of Mathematical Functions. With. Formulas, Graphs, and Mathematical Tables. Edited by M. Abramowitz and I. A. Stegun (RH Romer, 1988).

[33] A. R. Liddle and. H. Lyth, Cosmological Inflation and Large-Scale Structure (Cambrige University Press, Cambrige, 2000).

[34] K. Bamba, S. Nojiri, and S. D. Odintsov, Phys. Rev. D 90, 124061 (2014) [arXiv:1410.3993].

[35] P. A. R. Ade et al. (Planck Collaboration), Astron. Astrophys. 571, A16 (2014) [arXiv:1303.5083, arXiv:1303.5082, arXiv:1303.5076].

[36] E. Komatsu et al. (WMAP Collaboration), Astrophys. J. Suppl. 192, 18 (2011) [arXiv:1001.4538].

[37] G. Hinshaw et al. (WMAP Collaboration), Astrophys. J. Suppl. 208, 19 (2013) [arXiv:1212.5226]. 